\title{
Down-regulation of osteopontin expression by RNA interference affects cell proliferation and chemotherapy sensitivity of breast cancer MDA-MB-231 cells
}

\author{
LI YANG, LING WEI, WEI ZHAO, XINGWU WANG, GANG ZHENG, \\ MEIZHU ZHENG, XIANRANG SONG and WENSHU ZUO \\ Breast Cancer Center, Shandong Cancer Hospital and Institute, Jinan 250117, P.R. China \\ Received September 3, 2011; Accepted November 14, 2011
}

DOI: $10.3892 / \mathrm{mmr} .2011 .679$

\begin{abstract}
To determine the effect of the down-regulation of osteopontin (OPN) expression on the sensitivity of breast cancer cells to doxorubicin, a chemotherapeutic drug, OPN shRNA was used to transfect the OPN-positive breast cancer cell line MDA-MB-231. The expression of OPN mRNA and protein was analyzed by RT-PCR and Western blotting, respectively. The cell cycle distribution and apoptosis were analyzed by flow cytometry. Transfected MDA-MB-231 cells were treated with different concentrations of doxorubicin. Growth of MDA-MB-231 cells and $\mathrm{IC}_{50}$ of doxorubicin were determined by MTT assay. Expression of OPN mRNA and OPN protein of cells transfected with OPN shRNA was significantly decreased when compared to the negative controls $(\mathrm{P}<0.05)$. The number of MDA-MB-231 cells in the $\mathrm{S}$ phase was significantly increased, from 22.77 to $43.67 \%$. Transfection increased apoptosis from 3.61 to $4.91 \%$. Furthermore, the proliferation of MDA-MB-231 cells transfected with OPN shRNA was significantly decreased when compared to the negative control (transfected with empty vector). Sensitivity of MDA-MB-231 cells to doxorubicin was enhanced after treatment with OPN shRNA. The $\mathrm{IC}_{50}$ values of doxorubicin of the OPN-transfected group, the MDA-MB-231 cells and the negative transfected group were $0.13588,0.83869$ and $0.79652 \mu \mathrm{g} / \mathrm{ml}$, respectively. Down-regulation of OPN significantly inhibits expression of OPN protein in MDA-MB-231 breast cancer cells, reduces cell proliferation and increases their sensitivity to doxorubicin.
\end{abstract}

\section{Introduction}

Breast cancer is the most common malignancy in women, with increasing incidences noted worldwide. Since breast cancer

Correspondence to: Professor Wenshu Zuo, Breast Cancer Center, Shandong Cancer Hospital and Institute, Jinan 250117, P.R. China E-mail: zws12346@yahoo.com.cn

Key words: breast neoplasms, osteopontin, RNA interference, chemotherapy sensitivity is relatively sensitive to chemotherapy, chemotherapy usually plays a crucial role in systemic treatments of breast cancer, which is not replaceable by other measures, such as surgery and radiation therapy. However, development of multi-drug resistance (MDR) decreases chemotherapy efficacy. Furthermore, tumors in patients usually have varying sensitivity to chemotherapy, especially the drug dosage level. Therefore, reducing the necessary dosage level and increasing chemotherapy efficacy become more and more important in clinics.

Osteopontin (OPN) is a secreted glycophosphoprotein expressed in a number of cell types, such as epithelial lineages and leukocytes. It is involved in multiple processes, including tumor growth, metastasis, angiogenesis and immune system regulation $(1,2)$. It regulates multiple cellular signal transduction pathways through binding to integrins $(3,4)$. OPN has been detected in primary breast tumors, with elevated expression in the plasma of patients with metastatic breast cancer $(5,6)$. OPN inhibits apoptosis of breast cancer cells and increases the migration of breast epithelial cells (7-12).

It has been reported that there is a relationship between OPN and chemotherapeutic drug resistance. Currently, studies are focused mainly on the relationship between high OPN expression and chemotherapeutic drug resistance (1,13-18). However, there are no reports concerning the influence of OPN silencing on the resistance of doxorubicin, a commonly used chemotherapeutic drug for the treatment of cancers of the breast, bladder, stomach, lung, ovaries, thyroid, soft tissue sarcoma and multiple myeloma.

RNA interference (RNAi) mediated by microRNAs (miRNAs) or siRNAs is often used in the inhibition of gene expression and research of gene functions. In this study, we found that RNAi down-regulates OPN expression and increases the sensitivity of the MDA-MB-231 breast cancer cell line to doxorubicin.

\section{Materials and methods}

Reagents. The MDA-MB-231 breast cancer cell line, which expresses OPN, was provided by the fundamental laboratory in the Cancer Research Institute of Shandong Province. OPN silenced cell line MDA-MB-343 was constructed in our laboratory. The- $\beta$-galactosidase staining kit was purchased from 
the Beijing Biyuntian Co. Fetal calf serum was purchased from the Hangzhou Evergreen Co. DMEM culture medium was purchased from Beijing Thermo Fisher Biology Co., Ltd. Rabbit anti-human OPN monoclonal antibody (Rabbit monoclonal to OPN) was purchased from Abcam Company. Doxorubicin was purchased from Qilu Pharmaceutical Co. Human breast cancer cell line MDA-MB-231 and OPN silenced cell line MDA-MB-343 were grown in DMEM medium containing $10 \%$ FBS.

Immunoblot analysis. Total protein was extracted using cell lysis buffer. The total protein concentration was measured by the Bradford method. The protein was separated on $10 \%$ SDS PAGE gels. Immunoblot analyses were performed using primary anti-OPN monoclonal antibody (1:200), antiactin antibody $(1: 10,000)$ and the secondary mouse antibody $(1: 10,000)$.

Flow cytometry. Cells $\left(10^{5}\right)$ in the logarithmic phase were collected and washed twice with PBS. After being fixed for $48 \mathrm{~h}$ at $4^{\circ} \mathrm{C}$ with $1 \mathrm{ml}$ of pre-cooled ethanol, the cells were washed twice with PBS and then resuspended in PBS. After adding $500 \mu \mathrm{l}$ of PI (protein inhibitor) and RNaseA and incubating for $1 \mathrm{~h}$, cell cycle and apoptosis were detected by flow cytometry.

Cell senescence test. Cells were washed once with PBS, and then fixed for $15 \mathrm{~min}$ at room temperature by adding $500 \mu \mathrm{l}$ of $\beta$-galactosidase staining solution. After removing the staining solution, $500 \mu 1$ dye solution was added, followed by incubation overnight at $37^{\circ} \mathrm{C}$. Cell staining was observed using microscopy. Qualitative analysis of cell senescence was performed.

$M T T$. Cells were placed in 96 -well plates $\left(4 \times 10^{3} /\right.$ well). After $24 \mathrm{~h}$, the DMEM medium was replaced with fresh medium. Then, $20 \mu \mathrm{l}$ of $5 \mathrm{mg} / \mathrm{ml}$ MTT was added at 14, 48, 72 and $96 \mathrm{~h}$, followed by incubation for $4 \mathrm{~h}$. The cells were collected by centrifugation, and then resolved in $150 \mu 1$ of DMSO, followed by incubation for $10 \mathrm{~min}$ with shaking. The absorbance (A) value at $490 \mathrm{~nm}$ was measured, and the cell proliferation curve was constructed.

Determination of cell proliferation inhibition rate (IR) was performed as follows: Cells in the transfected group, nontransfected group and negative transfected group were treated with a series of different concentrations of doxorubicin $(0,0.2$, $0.4,0.8,1.6$ and $3.2 \mu \mathrm{g} / \mathrm{ml}$ ) for $48 \mathrm{~h}$. The A value was measured using the same method as mentioned above.

IR was calculated as follows: IR $=\left(1-\mathrm{A}\right.$ value $_{\text {test group }} /$ A value $_{\text {control group }}$ ) $100 \%$. The half inhibition concentration $\left(\mathrm{IC}_{50}\right)$ of doxorubicin was also calculated.

Statistical analysis. The experimental data are expressed as the means \pm standard error (SEM). Statistical software (SPSS 12.0) was used for independent sample t-tests, followed by the one-way analysis of variance. P-value $<0.05$ was considered to denote a significant difference.

\section{Results}

Down-regulated expression of OPN in MDM-MB-343 cells. The OPN shRNA plasmid and the control shRNA vector were

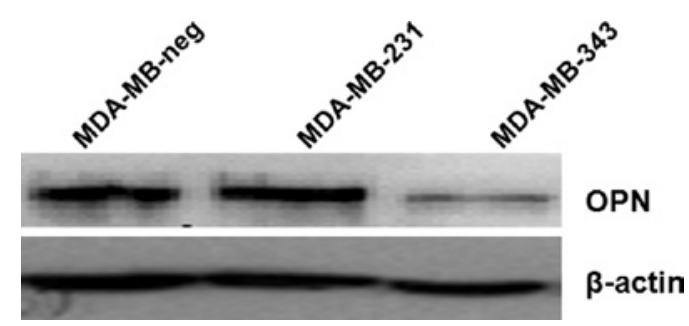

Figure 1. Determination of OPN levels in MDA-MB-231, MDA-MB-343 and MDA-MB-neg cells using immunoblot analyses. Total protein was extracted and separated on $10 \%$ SDS PAGE gels. Primary anti-OPN monoclonal antibody (1:200), anti-actin antibody $(1: 10,000)$ and the secondary mouse antibody $(1: 10,000)$ were used.

transfected into MDA-MB-231 cells to create an OPN silenced cell line, named MDA-MB-343. The control shRNA vectortransfected MDA-MB-231 cell line was named MDA-MB-neg. The OPN expression levels in MDA-MB-neg, MDA-MB-231 and MDA-MB-343 cells were determined using immunoblot analysis, with $\beta$-actin serving as the loading control. As shown in Fig. 1, expression of OPN protein in the MDA-MB-343 cells was significantly lower that that in the MDA-MB-231 and MDA-MB-neg cells, suggesting that OPN expression was indeed significantly reduced in the constructed MDM-MB-343 cells.

OPN gene silencing results in S phase arrest. Flow cytometric analysis of the three types of cells showed that the ratios of MDA-MB-231, MDA-MB-neg and MDA-MB-343 cells in the S phase were 21.10, 22.77 and $43.67 \%$, respectively (Fig. 2). These results suggested that OPN shRNA transfection causes a significant $S$ phase arrest of cells. The apoptotic rate of MDA-MB-neg was $3.61 \%$, while that of MDA-MB-343 was $4.91 \%$, suggesting that OPN down-regulation affects apoptosis slightly, if any (Fig. 3).

OPN gene down-regulation increases cell senescence. Cell senescence is a mechanism often related to tumor development. $\beta$-galactosidase staining was used to detect the cell senescence of MDA-MB-neg and MDA-MB-343 cells (Fig. 4). As shown in Fig. 4, OPN down-regulation increases cell senescence.

OPN gene down-regulation decreases cell proliferation. Proliferation of MDA-MB-231, MDA-MB-neg and MDA-MB-343 cells was measured using the MTT method at time points including 24, 48, 72 and $96 \mathrm{~h}$. As shown in Fig. 5, when compared to MDA-MB-231 and MDA-MB-neg cells, the growth of MDA-MB-343 cells was significantly reduced. Therefore, these results suggest that OPN gene downregulation decreases cell proliferation.

Down-regulation of the OPN gene increases the sensitivity of breast cancer cells to doxorubicin. To determine whether down-regulation of OPN affects the sensitivity of the MDA-MB-231, MDA-MB-neg and MDA-MB-343 cells to doxorubicin, these cells were treated with different concentrations of doxorubicin $(0.2,0.4,0.8,1.6$ and $3.2 \mu \mathrm{g} /$ $\mathrm{ml}$ ) for $48 \mathrm{~h}$, followed by cell proliferation measurements using the MTT assay. The absorbance value at $490 \mathrm{~nm}$ was 

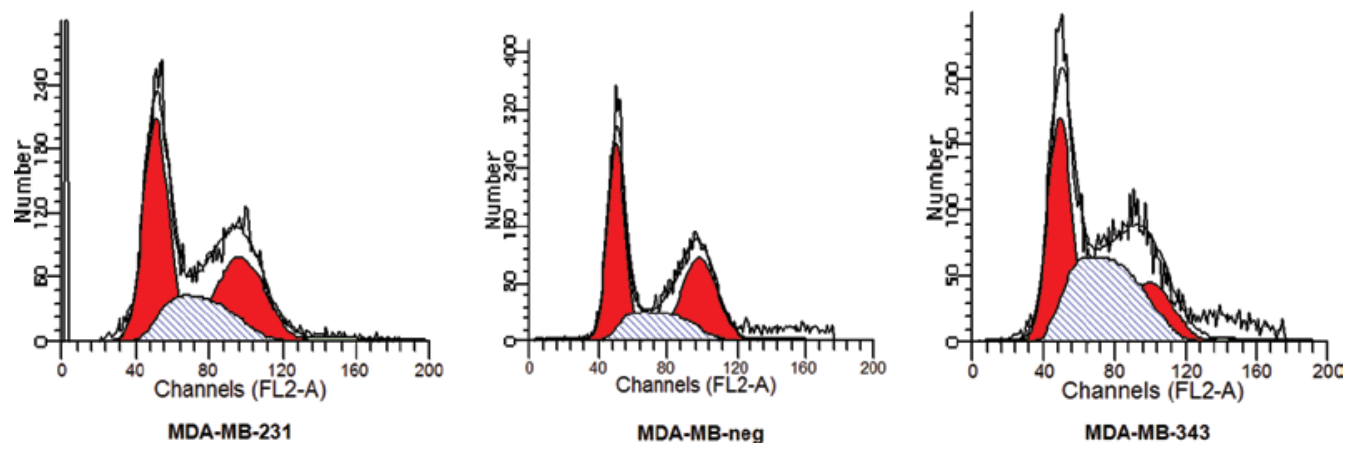

Figure 2. OPN shRNA transfection causes a significant $\mathrm{S}$ phase arrest of cells. Flow cytometric analysis of the three types of cells showed that the ratios of MDA-MB-231, MDA-MB-neg and MDA-MB-343 cells in the S phase were 21.10, 22.77 and 43.67\%, respectively.

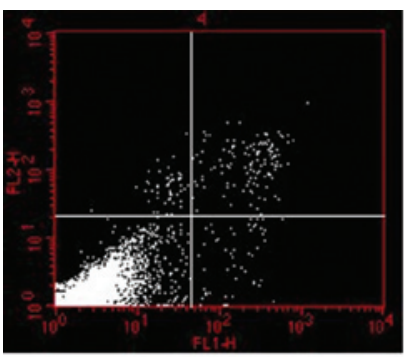

MDA-MB-neg

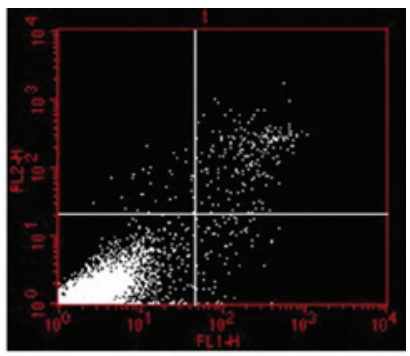

MDA-MB-343

Figure 3. Detection of the apoptotic rate of MDA-MB-neg and MDA-MB 343 cells. The apoptotic rate of MDA-MB-neg and MDA-MB-343 cells was detected using flow cytometric analysis.

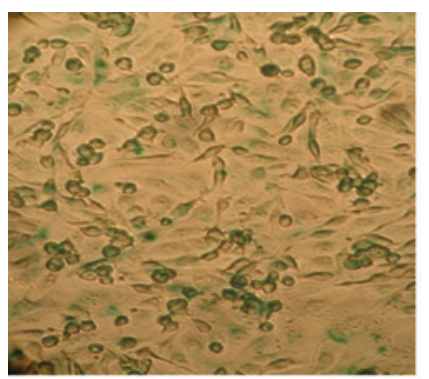

MDA-MB-neg

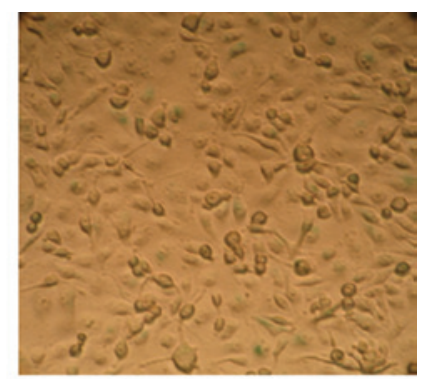

MDA-MB-343
Figure 4. Cell senescence test of the MDA-MB-neg and MDA-MB-343 cells. $\beta$-galactosidase solution was used to stain the cells. Cell staining was observed by microscopy. Qualitative analysis of the cell senescence was performed.

measured and the cell proliferation curve was constructed. Determination of cell proliferation IR was performed as described in Materials and methods.

As shown in Fig. 6, doxorubicin at concentrations of 0.4, $0.8,1.6$ and $3.2 \mu \mathrm{g} / \mathrm{ml}$ resulted in more effective proliferation inhibition of the MDA-MB-343 cells than that in the MDA-MB231 and MDA-MB-neg cells $(\mathrm{P}<0.01)$. The doxorubicin $\mathrm{IC}_{50}$ of the MDA-MB-231, MDA-MB-neg and MDA-MB-343 groups was $0.83869,0.79652$ and $0.13588 \mu \mathrm{g} / \mathrm{ml}$, respectively. When compared to the MDA-MB-231 and MDA-MB-neg cells, down-regulation of OPN in the MDA-MB-343 cells resulted in an $\sim 6$-fold decrease in the doxorubicin $\mathrm{IC}_{50}$ value, suggesting that down-regulation of OPN increases the sensitivity of the cells to doxorubicin.

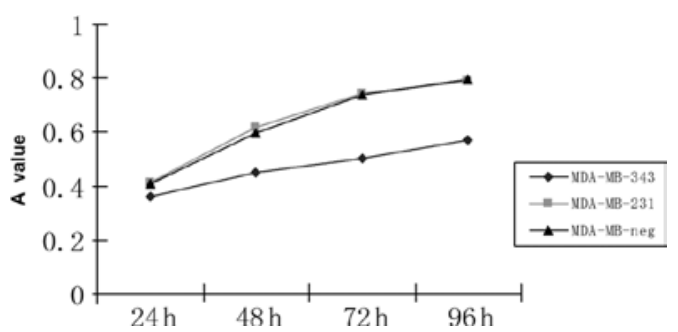

Figure 5. OPN gene down-regulation decreases cell proliferation. Proliferation of MDA-MB-231, MDA-MB-neg and MDA-MB-343 cells was measured using the MTT method at time points including 24, 48, 72 and $96 \mathrm{~h}$.

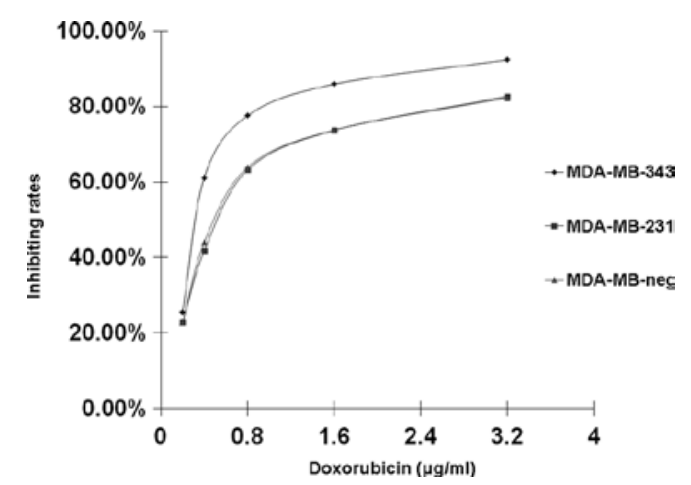

Figure 6. Down-regulation of the OPN gene increased the sensitivity of breast cancer cells to doxorubicin. The MDA-MB-231, MDA-MB-neg and MDA-MB-343 cells were treated with different concentrations of doxorubicin $(0.2,0.4,0.8,1.6$ and $3.2 \mu \mathrm{g} / \mathrm{ml})$ for $48 \mathrm{~h}$, followed by cell proliferation measurements using the MTT assay. The absorbance values at $490 \mathrm{~nm}$ were measured and the cell proliferation curve was constructed. Detection of cell proliferation inhibition rate (IR) was performed as described in Materials and methods.

\section{Discussion}

OPN is a secreted phosphorylated glycoprotein with multifunctions, playing important roles in tumor development, particularly in tumor invasion and metastasis. In this study, OPN expression of the MDA-MB-231 breast cell line was down-regulated by RNAi, and its effect on the cell sensitivity to doxorubicin was investigated. It was demonstrated by immunoblot analysis and RT-PCR that the OPN expression in the MDA-MB-343 cells was significantly reduced. Downregulation of OPN significantly inhibited expression of OPN 
protein in MDA-MB-231 breast cancer cells, reduced cell proliferation and increased their sensitivity to doxorubicin.

The senescence test results showed that OPN down-regulation increased cell senescence, which is consistent with the results reported by Shevde et al (19). The three groups of cells, including MDA-MB-231, MDA-MB-neg and MDA-MB-343 cells, were treated with different concentrations of doxorubicin. The doxorubicin $\mathrm{IC}_{50}$ value of the MDA-MB-343 group was $0.13588 \mu \mathrm{g} / \mathrm{ml}$, while the doxorubicin $\mathrm{IC}_{50}$ of the MDA-MB231 and MDA-MB-neg groups was 0.83869 and $0.79652 \mu \mathrm{g} /$ $\mathrm{ml}$, suggesting that down-regulation of OPN increases the sensitivity of the cells to doxorubicin.

Previous reports indicate that when OPN binds to the integrin $\alpha \beta 1$ (an extracellular matrix ligand), the PI-3K/Akt pathway is activated, leading to generation of resistance to chemotherapy (20-23). OPN interference inhibits the activation of the PI-3K/Akt signaling pathway, so that the sensitivity of cells to chemotherapeutic drugs increases. In addition, $S$ phase arrest in the cell cycle and decreases in cell proliferation were found upon down-regulation of OPN in our study. However, the specific mechanism remains unclear and should be further investigated. Doxorubicin is one of the most commonly used chemotherapy drugs for treating breast cancers. Yet, one side effect is cardiac toxicity, which is due to the cumulative dose of the drug. Our finding that the sensitivity of breast cancer cells to doxorubicin significantly increased when OPN expression was down-regulated suggests that the drug dose can be reduced when OPN expression is decreased. By this manner, the cumulative dose of drugs would be largely reduced, which will be helpful for the treatment with doxorubicin of breast cancer patients.

In conclusion, the present study demonstrates that RNAi effectively inhibits OPN expression, which is beneficial to breast cancer gene therapy. Our results indicate that OPN also inhibits breast cancer cell proliferation and facilitates cell senescence, resulting in a synergistic effect in combination with doxorubicin. Therefore, our study provides a basis for continued research for the treatment of breast cancer.

\section{Acknowledgements}

This study was supported by the Natural Foundation of Shandong Province (Y2008C167).

\section{References}

1. Beausoleil MS, Schulze EB, Goodale D, Postenka CO and Allan AL: Deletion of the thrombin cleavage domain of osteopontin mediates breast cancer cell adhesion, proteolytic activity, tumorgenicity, and metastasis. BMC Cancer 11: 25, 2011.

2. Denhardt D, Lopez C, Rollo E, Hwang S, An X and Walther S: Osteopontin-induced modifications of cellular functions. Ann NY Acad Sci 760: 127-142, 1995.

3. Zhang Z, Vuori K, Reed JC and Ruoslahti E: The alpha 5 beta 1 integrin supports survival of cells on fibronectin and up-regulates Bcl-2 expression. Proc Natl Acad Sci USA 92: 6161-6165, 1995.

4. Ding Q, Stewart JJ, Prince C, et al: Promotion of malignant astrocytoma cell migration by osteopontin expressed in the normal brain: differences in integrin signaling during cell adhesion to osteopontin versus vitronectin. Cancer Res 62: 5336-5343, 2002.
5. Senger D, Perruzzi C, Gracey C, Papadopoulos A and Tenen D: Secreted phosphoproteins associated with neoplastic transformation: close homology with plasma proteins cleaved during blood coagulation. Cancer Res 48: 5770-5774, 1988.

6. Singhal H, Bautista D, Tonkin K, O'Malley F, Tuck A, Chambers AH and Harris JFR: Elevated plasma osteopontin in metastatic breast cancer associated with increased tumor burden and decreased survival. Clin Cancer Res 3: 605-611, 1997.

7. Damiano JS, Cress AE, Hazlehurst LA, Shtil AA and Dalton WS: Cell adhesion mediated drug resistance (CAM-DR): role of integrins and resistance to apoptosis in human myeloma cell lines. Blood 93: 1658-1667, 1999.

8. Behera R, Kumar V, Lohite K, Karnik S and Kundu GC: Activation of JAK2/STAT3 signaling by osteopontin promotes tumor growth in human breast cancer cells. Carcinogenesis 31: 192-200, 2010.

9. Noti JD: Adherence to osteopontin via $\alpha v \beta 3$ suppresses phorbol ester-mediated apoptosis in MCF-7 breast cancer cells that overexpress protein kinase C- $\alpha$. Int J Oncol 17: 1237-1243, 2000.

10. He B, Mirza M and Weber GF: An osteopontin splice variant induces anchorage independence in human breast cancer cells. Oncogene 25: 2192-2202, 2006.

11. Hedley BD, Welch DR, Allan AL, et al: Downregulation of osteopontin contributes to metastasis suppression by breast cancer metastasis suppressor 1. Int J Cancer 123: 526-534, 2008.

12. Wang S, Liu Q, Zhang Y, et al: Suppression of growth, migration and invasion of highly-metastatic human breast cancer cells by berbamine and its molecular mechanisms of action. Mol Cancer 8: 81, 2009.

13. Hahnel A, Wichmann H, Kappler M, Kotzsch M, Vordermark D, Taubert $\mathrm{H}$ and Bache M: Effects of osteopontin inhibition on radiosensitivity of MDA-MB-231 breast cancer cells. Radiat Oncol 5: 82, 2010.

14. Ihnen M, Wirtz RM, Kalogeras KT, et al: Combination of osteopontin and activated leukocyte cell adhesion molecule as potent prognostic discriminators in HER 2- and ER-negative breast cancer. Br J Cancer 103: 1048-1056, 2010.

15. Das S, Samant RS and Shevde LA: Hedgehog signaling induced by breast cancer cells promotes osteoclastogenesis and osteolysis. J Biol Chem 286: 9612-9622, 2011.

16. El-Tanani MK, Yuen HF, Shi Z, et al: Osteopontin can act as an effector for a germline mutation of BRCA1 in malignant transformation of breast cancer-related cells. Cancer Sci 101: 1354-1360, 2010.

17. Mi Z, Bhattacharya SD, Kim VM, Guo H, Talbot LJ and Kuo PC: Osteopontin promotes CCL5-mesenchymal stromal cell-mediated breast cancer metastasis. Carcinogenesis 32: 477-487, 2011.

18. Gu T, Ohashi R, Cui R, et al: Osteopontin is involved in the development of acquired chemo-resistance of cisplatin in small cell lung cancer. Lung Cancer 66: 176-183, 2009.

19. Shevde LA, Samant RS, Paik JC, et al: Osteopontin knockdown suppresses tumorigenicity of human metastatic breast carcinoma, MDA-MB-435. Clin Exp Metastasis 23: 123-133, 2006.

20. Aoudjit $F$ and Vuori K: Integrin signaling inhibits paclitaxelinduced apoptosis in breast cancer cells. Oncogene 20: 4995-5004, 2001.

21. Graessmann M, Berg B, Fuchs B, Klein A and Graessmann A: Chemotherapy resistance of mouse WAP-SVT/t breast cancer cells is mediated by osteopontin, inhibiting apoptosis downstream of caspase-3. Oncogene 26: 2840-2850, 2007.

22. Allan AL, George R, Vantyghem SA, et al: Role of the integrinbinding protein osteopontin in lymphatic metastasis of breast cancer. Am J Pathol 169: 233-246, 2006.

23. Liang K, Lu Y, Li X, Zeng X, Glazer RI, Mills GB and Fan Z: Differential roles of phosphoinositide-dependent protein kinase-1 and akt1 expression and phosphorylation in breast cancer cell resistance to paclitaxel, doxorubicin, and gemcitabine. Mol Pharmacol 70: 1045-1052, 2006. 